1 Universidade Federal da Bahia (UFBA), Instituto de Saúde Coletiva (ISC) Salvador (BA), Brasil. Orcid: https://orcid. org/0000-0001-52689450

laravbarros@gmail.com

2 Universidade Federal da Bahia (UFBA), Instituto de Humanidades, Artes e Ciências Professor Milton Santos (IHAC) - Salvador (BA), Brasil.

Orcid: https://orcid. org/0000-0002-80809146

carment@ufba.br

\section{Movimento dos Trabalhadores Rurais Sem Terra e saúde do campo: revisão integrativa do estado da arte}

\author{
Landless Workers Movement and rural healthcare: integrative review \\ of the state of art
}

Larissa Daiane Vieira Barros ${ }^{\mathbf{1}}$, Carmen Fontes Teixeira ${ }^{\mathbf{2}}$

DOI: $10.1590 / 0103-11042018 S 227$

RESUMO O objetivo do trabalho foi caracterizar a produção científica acerca do Movimento dos Trabalhadores Rurais Sem Terra (MST) até 2016, com ênfase na identificação e na análise dos trabalhos que tratem do tema 'saúde', buscando identificar os problemas e a necessidade de saúde da população vinculada ao MST, bem como as concepções, projetos e práticas de saúde desse Movimento. Trata-se de uma revisão integrativa da literatura, que analisou 108 trabalhos capturados nas bases BVS e Capes, sendo que, destes, 15 tratam especificamente das questões de saúde. Os resultados evidenciam a precariedade das condições de vida e de saúde das populações acampadas e assentadas e revelam a multiplicidade de problemas e necessidades de saúde não atendidas de grupos específicos, a exemplo dos trabalhadores, mulheres e crianças. Observa-se, entre as lideranças do Movimento, uma concepção abrangente de Saúde e uma diversidade de posições com relação à participação do MST nas instâncias de controle social do SUS. Conclui-se apontando a necessidade de aprofundamento dos estudos sobre a capacidade de o movimento sanitário incorporar os movimentos do campo em sua base de sustentação, principalmente se o MST tem sido um aliado no processo de Reforma Sanitária Brasileira.

PALAVRAS-CHAVE Reforma dos serviços de saúde. Sistema Único de Saúde. Saúde da população rural.

ABSTRACT This work aims to characterize the scientific material which has been produced about the Landless Workers Movement (MST) until 2016, with emphasis on the identification and analysis of those works that deal with the topic of 'Health'. This work also strives to identify this Movement's health practices, conceptions, and projects. This is an integrative review of the literature related to the issue at hand. Out of the 108 works analyzed, which were found in the BVS and Capes databases, 15 specifically dealt with health issues. The results show that due to the precariousness of the living conditions in the settlements, the residents, such as workers, women and children, were found to have numerous of their needs and health problems unaddressed. One can observe that among the Movement's leaders there is a comprehensive understanding of Health as well as different perceptions regarding the participation of the MST in the instances of social control of the Brazilian Unified Health System (known as SUS). The author concludes by directing the reader's attention to the fact that this is a topic that is still lacking more in-depth research. More studies need to be done on the movement, especially those that will further analyze the relationship of the MST and the Brazilian Health Reform.

KEYWORDS Health care reform. Unified Health System. Rural health. 


\section{Introdução}

O Movimento dos Trabalhadores Rurais Sem Terra (MST), principal movimento social do campo no Brasil e um dos mais importantes no mundo, foi criado em 1984 tendo como principais objetivos a luta pela terra, pela reforma agrária e por uma sociedade justa e fraterna ${ }^{1,2}$. Ao longo do período em que tem atuado, o MST garantiu a conquista da terra para mais de 350 mil famílias, sendo que, atualmente, esse movimento está presente em 24 estados brasileiros, contando com uma base social que abarca mais de 1,5 milhão de pessoas ${ }^{\mathbf{1 , 3}, 4}$.

Dadas as características desse movimento, que contempla o deslocamento de grupos populacionais por grandes extensões do território nacional, bem como a montagem de acampamentos e assentamentos precários, é importante investigar a problemática de saúde enfrentada pela população envolvida, tanto do ponto de vista das suas condições de saúde quanto do ponto de vista do acesso às ações e serviços destinados ao atendimento de suas necessidades de saúde. Além disso, considerando que se trata de um movimento social portador de um projeto político que ultrapassa a dimensão meramente econômica, de luta pelos meios de produção, especificamente a terra, cabe investigar quais as propostas e os projetos específicos com relação a outras dimensões do processo de reprodução social, principalmente a garantia dos direitos, entre os quais, o direito à saúde.

Nesse sentido, cabe, em primeiro lugar, realizar uma revisão da literatura existente sobre este movimento, buscando identificar os temas que têm se tornado objetos de investigação pela comunidade científica e os estudos que abordem notadamente a questão da saúde. Este trabalho se insere em um projeto de pesquisa mais amplo, que trata de analisar as concepções e práticas de saúde desenvolvidas no âmbito do MST, buscando compreender sua aproximação ou distanciamento com os princípios, diretrizes e estratégias propostas pelo movimento da Reforma Sanitária Brasileira ${ }^{5}$ (RSB), movimento que colocou na agenda política do Estado brasileiro a questão do direito universal à saúde, aprovado na Constituição Federal de $1988^{6}$, base jurídica do difícil processo de construção do Sistema Único de Saúde (SUS) nos últimos 30 anos $7 \mathbf{8}$.

Trata-se de investigar, portanto, até que ponto o MST pode ser visto como um potencial aliado nas lutas pela RSB e pela construção do SUS no Brasil ou se suas propostas apontam para outra direção. Nessa perspectiva, foram definidas algumas questões norteadoras da revisão bibliográfica, quais sejam: Quais as características da produção científica sobre o MST? Quais os principais temas investigados? Como é abordada a saúde no âmbito desta produção?

Desse modo, o objetivo do trabalho é caracterizar a produção científica acerca do MST até 2016, com ênfase na identificação e análise dos trabalhos que tratem do tema 'saúde', buscando identificar os problemas e necessidade de saúde da população vinculada ao MST, bem como as concepções, projetos e práticas de saúde deste Movimento.

\section{Estratégia metodológica}

O desenho deste estudo contempla uma revisão bibliográfica integrativa, um tipo de revisão que busca sistematizar o conhecimento científico disponível, aproximando o pesquisador da problemática que deseja apreciar, possibilitando conhecer a evolução do tema ao longo do tempo e visualizar oportunidades/lacunas/questões de pesquisa9. Assim, buscamos realizar uma análise e síntese do conhecimento científico produzido sobre o MST mediante a aplicação de métodos explícitos e sistematizados de busca, síntese da informação e análise crítica da produção encontrada.

Inicialmente, tentamos definir os descritores de busca no site do Descritores em 
Ciências da Saúde (DeCS). Foram utilizados termos como: 'população do campo'; 'saúde da população do campo'; 'movimento campesino'; 'movimento agrário'; 'movimento sem terra'; 'movimento dos trabalhadores rurais sem terra', mas nenhum desses descritores foi encontrado. Nesse sentido, optamos pelo uso do termo 'movimento dos trabalhadores rurais sem terra', buscando realizar uma busca avançada e garantindo o acesso aos artigos que contivessem essa expressão no título e/ou nas palavras-chave. Realizamos a busca em duas plataformas, a Biblioteca Virtual em Saúde (BVS) ${ }^{10}$ (http://brasil.bvs. br/) e o Banco de Periódicos Capes ${ }^{\mathbf{1 1}}$ (http:// www.periodicos.capes.gov.br/), por disponibilizarem um amplo acervo de trabalhos indexados nessas bases.

Conforme a figura 1 , a consulta às bases de dados foi realizada no dia 9 de junho de 2017, tendo sido encontrado um total de 385 trabalhos, cujos títulos foram lidos de modo a excluir repetições. Em seguida, foram lidos os resumos, aplicando como critérios de inclusão os trabalhos que apresentavam informações e discussões sobre o MST, não sendo considerados os artigos que discutem reforma agrária, assentamentos e acampamentos sem referência ao MST. Desse modo, foram selecionados 99 artigos, 6 dissertações e 3 teses publicados até o ano de 2016, em periódicos revisados por pares, disponíveis integralmente e nas línguas portuguesa, inglesa e espanhola.

Figura 1. Processo de seleção dos artigos analisados

Data da busca

Base de dados

Encontrados

Selecionados

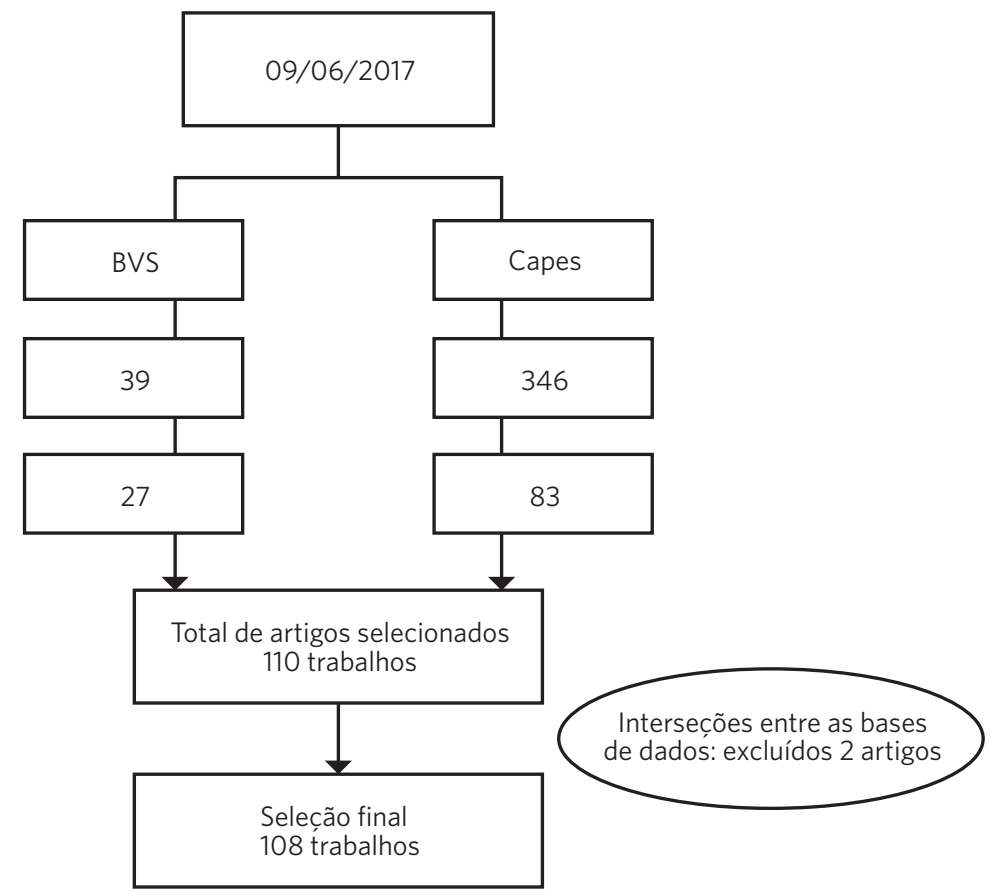


Os resumos dos trabalhos selecionados foram novamente lidos de forma a extrair um conjunto de informações: o tipo de trabalho, idioma, tema abordado, ano de publicação e procedência institucional dos autores. Essas informações quantitativas foram processadas para construir tabelas e gráficos que apresentam as características gerais desta produção. Especificamente com relação ao tema abordado, os trabalhos foram agrupados em sete categorias, quais sejam:

a) MST e Reforma Agrária: inclui trabalhos que abordam a estrutura fundiária brasileira e a participação do MST nas lutas pela Reforma Agrária no Brasil;

b) MST e Educação do Campo: trabalhos que discutem as Políticas de Educação para o Campo e os princípios e práticas educativas do MST;

c) MST como Ator Político: aborda o MST enquanto ator político importante no cenário de lutas no Brasil e na América Latina, seus processos organizativos e discursos políticos; d) MST e Mídia: compreende estudos sobre visões, representações e discursos da mídia brasileira sobre o MST;

e) MST e Gênero: inclui artigos que analisam a divisão sexual do trabalho e as desigualdades de gênero dentro do MST, bem como a participação das mulheres nos processos decisórios do movimento;

f) MST e Saúde: artigos e dissertações que analisam as condições de vida e de trabalho no campo, concepções do processo saúde-doença e suas práticas de saúde, formas de participação e organização na saúde e as políticas de saúde para o campo;

g) Outros: trabalhos que não se enquadravam nas categorias acima delimitadas e que abordam temas como: biblioteca virtual do MST, cooperativismo rural etc.

Conforme pode ser visualizado na tabela 1, dos 108 trabalhos selecionados, 29,62\% referem-se ao MST/ator político, e 22,22\%, ao MST/Reforma Agrária, sendo que apenas 13,88\% (15) dos estudos são da área de saúde e foram publicados entre 2007 e 2015.

Tabela 1. Distribuição dos trabalhos segundo tema e tipo

\begin{tabular}{lrrrrrrrr}
\hline \multirow{2}{*}{ Categoria temática } & \multicolumn{2}{c}{ Artigos } & \multicolumn{2}{c}{ Dissertações } & \multicolumn{2}{c}{ Teses } & \multicolumn{2}{c}{ Total de trabalhos } \\
\cline { 2 - 8 } MST/Educação do Campo & No & $\%$ & № & $\%$ & № & $\%$ & № & $\%$ \\
MST/Reforma Agrária & 12 & $100 \%$ & - & - & - & - & 12 & $11,11 \%$ \\
MST/Ator Político & 24 & $100 \%$ & - & - & - & - & 24 & $22,22 \%$ \\
MST/Mídia & 28 & $90,62 \%$ & 1 & $3,1 \%$ & 3 & $9,37 \%$ & 32 & $29,62 \%$ \\
MST/Saúde & 5 & $83,33 \%$ & 1 & $16,6 \%$ & - & - & 6 & $5,55 \%$ \\
MST/Gênero & 13 & $86,66 \%$ & 2 & $13,33 \%$ & - & - & 15 & $13,88 \%$ \\
MST/Outros & 5 & $83,33 \%$ & 1 & $16,6 \%$ & - & - & 6 & $5,55 \%$ \\
\hline Total & 12 & $92,3 \%$ & 1 & $7,69 \%$ & - & - & 13 & $12,03 \%$ \\
\hline Fonte: Elaboração própria. & $\mathbf{9 9}$ & $\mathbf{9 1 , 6 6 \%}$ & $\mathbf{6}$ & $\mathbf{5 , 5 5 \%}$ & $\mathbf{3}$ & $\mathbf{2 , 7 7 \%}$ & $\mathbf{1 0 8}$ & $\mathbf{1 0 0} \%$ \\
\hline
\end{tabular}


Finalmente, os trabalhos incluídos na categoria MST e Saúde (13 artigos e 2 dissertações) foram lidos na íntegra e submetidos a uma análise de conteúdo temática ${ }^{12,13}$, tratando-se de sistematizar as principais contribuições desses estudos ao conhecimento dos problemas, necessidades e demandas de saúde da população diretamente envolvida com o MST, bem como as propostas e soluções encontradas com relação ao acesso às ações e serviços de saúde.

\section{Resultados}

Os 15 trabalhos que tratam de questões de saúde no MST foram produzidos, em sua totalidade, por pesquisadores brasileiros, em sua maioria, vinculados às universidades da região Sudeste e Centro-Oeste, tendo sido publicados em periódicos da área de saúde pública/coletiva no período entre 2007 e 2015. Resultaram de pesquisas empíricas realizadas em assentamentos e acampamentos do MST, utilizando metodologias qualitativas, especialmente entrevistas, observação, grupo focal e análise documental.

Dois dos trabalhos, em particular, foram feitos com o Coletivo Nacional de Saúde do Movimento ${ }^{4,14}$, entidade que congrega representações de lideranças do MST de todos os estados do País. Entre os trabalhos, seis citam a RSB4,15,18; e apenas um, a Reforma Psiquiátrica ${ }^{16}$, explicitando a defesa do MST dos princípios do SUS e da saúde como conquista popular e dever do Estado.

Ressalta-se a presença dos princípios da educação popular em saúde $4,15,16,19,20,23,24$ enquanto referencial e método de análise do MST, principalmente a produção coletiva de saberes, as práticas de cuidado e os processos de luta do movimento. Isso pode estar relacionado com a forte influência do pensamento de Paulo Freire na construção da pedagogia do movimento ${ }^{23}$, pela correspondência de princípios e valores educativos presentes tanto na educação popular em saúde quanto nas formas de organização política do MST, como também pela vinculação teórico-política dos autores dos trabalhos e suas relações com os Sem-Terra. Outro ponto relevante é a discussão sobre a saúde das mulheres do campo 15,22,24,25, o que demonstra certo avanço no debate sobre as questões de gênero e sobre sua relação com a saúde das mulheres do campo, sem que, entretanto, sejam identificados artigos que abordem a questão étnico-racial.

\section{Análise temática dos trabalhos sobre MST e Saúde}

Todos os trabalhos analisados tratam, em maior ou menor grau, das condições de saúde da população que vive nos assentamentos e acampamentos do MST, apontando uma diversidade de problemas que afetam grupos específicos dessa população, a exemplo das condições de trabalho no campo $^{\mathbf{2}, 15,20,22,24,26,27}$, infraestrutura e a problemática da saúde da mulher ${ }^{15,22,26,27}$. Chama atenção a lacuna existente com relação à situação de saúde das crianças, ainda que existam informações pontuais em alguns trabalhos analisados 2,17,20,27. Além das condições de saúde, grande parte dos trabalhos relata a problemática da acessibilidade 2,14,15,17,19,20,22,24,25,27 da população assentada/acampada às ações e serviços do sistema de saúde, apontando dificuldades no acesso; em contrapartida, eles analisam as concepções e práticas de saúde 2,14-17,19,27 realizadas nos acampamentos e assentamentos.

A leitura detalhada de cada um desses trabalhos permitiu a sistematização dos conhecimentos produzidos, o que possibilitou a identificação dos principais problemas enfrentados por essa população, como segue.

\section{O trabalho e a saúde no campo}

Os estudos revelam que o trabalho no campo é marcado por longas e extenuantes jornadas de trabalho, longos períodos de deslocamento, 
predomínio do trabalho braçal e repetitivo e exposição aos diversos fenômenos da natureza que podem significar riscos à saúde. Entre os principais agravos à saúde 2,20,23,24,27 ocasionados pelo trabalho rural, estão a intoxicação pelo uso de agrotóxicos, excessiva exposição à radiação solar, dores musculares e esgotamento físico, acidentes com equipamentos cortantes e com animais peçonhentos. As melhores condições de trabalho estão nos assentamentos, em detrimento dos acampamentos e do trabalho sazonal assalariado, a exemplo do corte da cana de açúcar por boias-frias,

afetando a saúde do trabalhador devido ao ritmo intenso e acelerado dos movimentos braçais, postura inadequada do corpo por longos períodos, reduzido período de descanso ${ }^{\mathbf{2 ( 1 5 9 9 )}}$.

As mulheres conjugam

atividades na lavoura, os afazeres da casa, o cuidado com as crianças e a militância na luta social24(592).

As trabalhadoras rurais produzem alimentos e garantem a subsistência da família desenvolvendo trabalhos no roçado, além de se ocuparem com o plantio de ervas medicinais e com o artesanato 22(1342).

A presença do trabalho infantil2o no campo é marcada pela divisão sexual do trabalho, na medida em que as meninas são submetidas ao trabalho doméstico muito cedo, enquanto os meninos são inseridos precocemente nas fazendas. Há casos de contaminação de crianças e mulheres pela exposição aos agrotóxicos, por meio do transporte dos produtos, pelo ar, pela água e pelo solo, pelo processo de higienização das roupas pelas mulheres ou mesmo pelas brincadeiras com resíduos e embalagens pelas crianças.

A intoxicação pelo uso dos agrotóxicos ${ }^{20}$ é apontada como principal agravo à saúde da população do campo, acarretando incapacidade para o trabalho devido ao longo período de internação, às dores de cabeça crônica, à disfunção e à perda de órgãos (fígado e rins), como também aos casos de suicídio, que podem estar associados à intoxicação crônica e às precárias condições de vida e de trabalho no campo ${ }^{27}$.

\section{Infraestrutura e saúde no Campo}

Vários estudos 2,16,19,20,26,27 revelam condições de moradia insalubres, baixa cobertura de saneamento básico, dificuldades de acesso à água potável (cacimbas e poços) para consumo e irrigação das lavouras, precariedade ou ausência de coleta de lixo, de energia elétrica e de transporte coletivo.

Constata-se que há uma

insuficiente oferta de serviços públicos e de infraestrutura, o que inclui serviços de saúde, saneamento, moradia, transporte, lazer e outros, bem como pela ausência de um modelo de atenção à saúde direcionado para a população rura|2(1596).

A infraestrutura produtiva, que inclui terras férteis, assistência técnica, ferramentas e aceso às políticas de crédito, também é bastante precária e se apresenta como condição básica de permanência na terra e produção de saúde no campo ${ }^{16,26,27}$.

\section{Perfil de morbidade}

Verificou-se alta incidência de doenças infectoparasitárias ${ }^{27}$, a exemplo da leishmaniose e da esquistossomose. Outros problemas de saúde $\mathbf{1 5 , 1 6 , 2 1 , 2 5}$ foram referidos pela população do campo, como: vômitos, diarreias, dores musculares, subnutrição, reumatismo, intoxicação por agrotóxicos, uso abusivo de álcool e outras drogas, assim como transtornos mentais leves e graves (ex.: depressão e suicídio).

\section{Saúde mental da população do campo}

O consumo prejudicial de álcool e outras 
drogas e os transtornos mentais 'leves e graves' são os problemas de saúde mental mais recorrentes nos assentamentos e acampamentos do MST $^{15}$. Constataram-se também

casos de maior incidência de sofrimento psicológico intenso, depressão e mesmo suicídios em grupos de trabalhadores expostos aos agrotóxicos 27(92-93).

\section{Saúde integral da mulher}

Dores lombares, cefaleia e depressão aparecem como principais problemas de saúde relatados pelas mulheres ${ }^{24}$. A condição de mulher do campo e do MST é marcada por relações desiguais de gênero, o que impõe muitas dificuldades de acesso às informações e ações de saúde. A atenção à saúde das mulheres do MST mostrou-se fragmentada, de baixa qualidade e definida pela condição reprodutiva. Não foram identificadas ações de promoção da saúde da mulher nem prevenção de agravos específicos nesse grupo populacional, a exemplo de câncer de mama, útero, diabetes, hipertensão, ou mesmo violência contra a mulher, prioridades na Política Nacional de Saúde Integral da Mulher ${ }^{28}$. Verifica-se, contudo, que a gravidez se constitui uma condição de saúde que possibilita o primeiro acesso aos serviços de pré-natal e, de certa forma, estimula a continuidade do cuidado, como também a realização de exames essenciais para as mulheres acampadas e assentadas ${ }^{26}$.

\section{Segurança alimentar e nutricional}

As famílias assentadas, acampadas e boias-frias apresentam diferentes condições de segurança alimentar e nutricional.

Os trabalhadores boias-frias apresentaram um alto índice de insegurança alimentar $(39,5 \%)$, quase o dobro da proporção entre as famílias acampadas e quatro vezes mais que

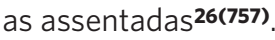

O acesso à terra, a criação de animais, o cultivo de alimentos destinados ao autoconsumo familiar, além da produção para geração de renda, constituem atividades fundamentais para o acesso regular e permanente aos alimentos de qualidade, condição essencial para segurança alimentar e produção de saúde 2,17,20,26,27. O uso da agroecologia $^{16,19,27}$, o sistema de trocas de alimentos e a vinculação ao $\mathrm{MST}^{\mathbf{2}, 20}$ aparecem também como centrais na produção de práticas alimentares promotoras de saúde e da segurança alimentar e nutricional no campo.

\section{Concepções e práticas de saúde do MST}

Os estudos sobre o MST apontam que o movimento adota uma concepção ampliada e abrangente de saúde ${ }^{2,14-17,19,27}$, que

envolve a relação indivíduo-sociedade-natureza, valorizando a organização política, a preservação ambiental, as práticas de saúde populares e a medicina tradiciona|2(1596).

Nessa perspectiva, saúde é entendida como a

capacidade de lutar contra tudo o que os agride e os ameaça, inclusive a doença, e é resultante das condições de alimentação, habitação, educação, renda, meio ambiente, trabalho, transporte, emprego, lazer, liberdade, acesso e posse da terra e acesso aos serviços de saúde ${ }^{17(495)}$.

Entre as principais práticas de saúde realizadas pelos assentamentos e acampamentos

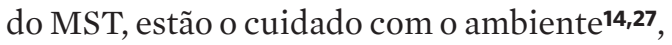
visitas domiciliares ${ }^{\mathbf{2}, 17}$, acompanhamento dos pacientes ${ }^{\mathbf{1 4}}$, dietas como formas de tratamentos ${ }^{22}$, práticas das parteiras ${ }^{22}$, crença religiosa ${ }^{22}$, produção, consumo e orientações para o uso de plantas medicinais, 14,16,17,19,22,27 (fitoterápicos), acesso aos serviços da rede de saúde ${ }^{14,27}$ e ações de educação ${ }^{14,17}$ e planejamento em saúde organizadas 
coletivamente pelo setor de saúde do movimento e pelos ACS, mas conta também com parcerias com as pastorais da criança e da saúde18. Identificaram-se ainda como práticas de cuidado "a terapia floral, a radiestesia, o passe espiritual e o reiki"15(1542).

Cabe enfatizar que o Coletivo Nacional de Saúde do MST, criado em 1998, cumpre papel importante na realização de encontros regulares para discussão de temas relevantes (DST/Aids, fitoterapia, políticas de saúde/ SUS, participação social etc.), organização do setor de saúde dos acampamentos e assentamentos e realização de cursos de formação no campo da saúde coletiva para seus integrantes ${ }^{\mathbf{1 4}}$, realizados na escola do MST - o Instituto Educacional Josué de Castro ${ }^{14}$, como também a sua contribuição na formulação de políticas de saúde para a população do campo $14,16,26,27$.

\section{Acesso aos serviços de saúde}

Para as famílias da população assentada e acampada, o "SUS não tem atendido as necessidades de saúde da maioria delas, principalmente pela dificuldade do acesso aos serviços"26(757). Como principais problemas que limitam o acesso, estão: "dificuldade de deslocamento até a cidade onde se concentra a oferta desses serviços e a dificuldade de atendimento"28(128), a "baixa resolutividade dos serviços oficias de saúde e pequena integração com as práticas em saúde utilizadas pelos assentados e acampados"19(441).

Relatos de discriminação pela condição de 'Sem-Terra'17, descontinuidade do atendimento, ausência de informações em saúde e insuficiência ou inexistência de equipes de saúde da família, filas nas unidades de saúde e dificuldades para realizar consultas e exames também foram identificados como fatores limitantes do acesso por essa população, o que contribui para a busca dos serviços tardiamente, apenas quando os sintomas se agravam, quando não são resolvidos com as práticas de saúde do movimento ou acessam o setor privado ${ }^{27}$ como alternativa de resolução.

Alguns artigos apontam que o Agente Comunitário de Saúde (ACS) $\mathbf{1 4 , 1 7 , 2 2}$ cumpre papel importante na garantia do acesso aos serviços de saúde, já que se trata de alguém da própria comunidade que estabelece o diálogo com as unidades de saúde e desenvolve atividades de promoção e educação em consonância com os valores, princípios e cultura do movimento. Quanto ao acesso a serviços de saúde mental,

os relatos das lideranças sobre as tentativas de acesso à atenção em saúde mental mostram que ainda são muitos os desafios à efetivação de uma rede de serviços substitutivos que atenda às populações assentadas e acampadas ${ }^{15(1543)}$, [especialmente nos municípios de pequeno porte].

\section{Ação política na área de saúde: pro- postas e estratégias}

O movimento está organizado em coletivos locais (assentamentos e acampamentos), estaduais e nacionais ${ }^{\mathbf{2 6 , 4}}$ que "atuam na promoção e controle de políticas públicas e articulação com outras entidades e movimentos"26(759). Os estudos que abordam a atuação do Coletivo Nacional de Saúde do MST evidenciam que este cumpre papel importante na organização e luta pelo direito à saúde no movimento ${ }^{14,16,26,27}$, orientados pelos seguintes princípios e valores:

Luta pela valorização da vida, o acesso ao conhecimento e à informação, a saúde como dever do Estado, a atenção integral, a prioridade à promoção e à prevenção, o respeito às diferenças culturais, e o fortalecimento das práticas não convencionais e, finalmente, a saúde como conquista de luta popular14(15).

Segundo essas análises, o MST reivindica um sistema de saúde universal que responda 
às necessidades e demandas das populações do campo e da cidade, "repudia a doença como fonte de acumulação de riqueza e aponta para prioridades de investimento em ações de prevenção e promoção da saúde"27(78). Assim, para o Coletivo Nacional, "a política de saúde do campo deve ser baseada no fortalecimento e consolidação do SUS e dos princípios da Reforma Sanitária"14(11).

No que se refere às estratégias de luta pelo direito à saúde, o MST considera que

a participação nas esferas instituídas de controle social do SUS não constitui a prioridade desse Coletivo, pois fazer o controle social do SUS significa, sobretudo, fazer a luta política por meio de estratégias de ocupação, de mobilizações e de marchas4(01).

Ou seja,

a luta pela saúde como direito integra essa agenda e busca denunciar as iniquidades em saúde vividas pelas populações do campo bem como valorizar as culturas, os saberes e as potencialidades dessas populações ${ }^{\mathbf{1 5 ( 1 5 3 8 )}}$.

Apesar do posicionamento apontado no estudo anterior com relação à participação do MST nas instâncias de controle social do SUS, foi possível identificar experiências de participação do MST em conselhos municipais e estaduais de saúde ${ }^{\mathbf{4 4 , 1 7}}$. Um dos artigos, inclusive, analisa essa diversidade de situações como decorrente das distintas ideias e perspectivas teóricas dos integrantes do MST sobre o 'estado burguês'4, contemplando, assim, uma vertente 'leninista', que rejeita a participação nos conselhos; e outra, mais 'gramsciana', que defende essa participação.

Além da controvérsia em torno da participação nos Conselhos de Saúde, um dos artigos14 analisa a participação do MST em outra instância de participação social em saúde, qual seja: o Grupo da Terra, espaço criado em 2004, vinculado ao Ministério da Saúde, que assegurou a participação de diversos movimentos sociais do campo, os quais, pela primeira vez, puderam participar diretamente do processo de discussão, decisão e construção de ações de saúde que respondam às suas necessidades. Com isso, segundo os autores, ocorreu a inclusão da população assentada na base de cálculo do Piso da Atenção Básica, a partir de

mudanças no financiamento e [...] incentivo à equidade, para estimular a criação de equipes do PSF em municípios pequenos com expressiva população rural14(14).

Dois dos trabalhos analisados citam a formulação e publicação de diversas políticas ${ }^{\mathbf{1 6}, 27}$, como a Política Nacional de Saúde do Trabalhador e da Trabalhadora ${ }^{29}$, Política Nacional de Práticas Integrativas e Complementares ${ }^{30}$, Política Nacional de Plantas Medicinais e Fitoterápicos ${ }^{31}$, a inclusão dos assentados no Programa Nacional de Habitação Rural ${ }^{32}$ e, particularmente, a Política Nacional de Saúde Integral das Populações do Campo e da Floresta ${ }^{33}$, indicando a entrada do tema da saúde da população do campo na agenda do Estado e a possibilidade de enfrentamento das iniquidades de saúde no espaço rural.

A mobilização política do MST em torno das questões de saúde, segundo alguns dos artigos analisados, conta com a parceria de diversos sujeitos, a exemplo das Pastorais da saúde e da criança, bem como de profissionais das Equipes de Saúde da Família (EqSF) comprometidos com a expansão e qualidade da Atenção Primária à Saúde: EqSF, algumas instituições de ensino, como Escola de Saúde Pública de Minas Gerais e universidades, especialmente em atividades de formação de lideranças que atuam no setor saúde do movimento. O Instituto Educacional Josué de Castro, escola do MST localizada em Veranópolis (RS) ${ }^{\mathbf{1 4}}$, constitui-se espaço de encontros e formação de seus atores no que se refere às temáticas relacionadas com a saúde. Verifica-se também o importante 
papel dos trabalhos de extensão e de residências multiprofissionais realizadas pelas universidades no espaço rural, contribuindo para a discussão e para a produção coletiva de conhecimentos sobre a saúde do campo, fortalecimento e valorização das práticas de cuidado em saúde em assentamentos e acampamentos do MST.

Por fim, cabe destacar que, no âmbito internacional, o MST, como um dos atores do campo na América do Sul, está articulado a diversos movimentos globais pela saúde ${ }^{\mathbf{1 8}}$, a exemplo do Movimento de Saúde dos Povos, Fórum Social Mundial de Saúde e o Fórum Social Mundial. No entanto, Carneiro ${ }^{14}$ considera a atuação do MST na saúde ainda incipiente, quando comparado com a diversidade de ações e capacidade de organização de outros setores do movimento. Aponta a falta de infraestrutura e de apoio econômico para a realização dos trabalhos, a pouca sistematização das experiências, o baixo reconhecimento da importância do setor saúde nas instâncias decisórias do MST como alguns elementos que determinam os limites das ações de saúde dentro do movimento.

\section{Discussão e conclusões}

Um primeiro aspecto a ser destacado do conjunto de trabalhos analisados é a diversidade de abrangência territorial e populacional na medida em que a maioria deles recorta realidades específicas em determinados estados e regiões do País. No entanto, os resultados da grande maioria desses trabalhos desvelam a precariedade das condições de vida e de saúde da população do campo, apontando a multiplicidade de problemas que afetam trabalhadores, mulheres, crianças que vivem nas condições dos acampamentos/assentamentos do MST. Esses achados convergem com os resultados apresentados pela Pesquisa sobre a qualidade de vida, produção e renda dos Assentamentos da reforma agrária ${ }^{34}$, coordenada pelo Instituto
Nacional de Colonização e Reforma Agrária (Incra), publicada em 2010, que revela que apenas $43,86 \%$ têm acesso regular à energia elétrica, $79 \%$ das famílias assentadas têm acesso suficiente à agua para consumo doméstico, $76 \%$ utilizam fossa e apenas $1,14 \%$ tem acesso à rede de esgoto. Além disso, 57\% dos assentados avaliam como péssimo ou ruim as condições das estradas e vias de acesso aos lotes e $56 \%$ avaliam como péssimo ou ruim o acesso aos postos de saúde e hospitais. Chama atenção, inclusive, o fato da melhoria das condições de vida das famílias assentadas quando comparadas às acampadas, o que demonstra a importância da garantia do direito à terra para a melhoria das condições de vida e de saúde da população do campo.

No que se refere especificamente às questões de saúde, chama atenção o consenso entre os autores de que o MST tem uma concepção ampliada de saúde, de certa forma coerente com os princípios e diretrizes da RSB e do SUS. No entanto, a situação epidemiológica da população do campo e os problemas de acesso aos serviços apontados nos trabalhos analisados evidenciam a persistência de iniquidades, que não estão sendo adequadamente enfrentadas pelas Políticas Públicas de Saúde, e revelam a insuficiência dos processos de implementação, que repercutem na baixa cobertura dos serviços, desarticulação das ações que resultam na incapacidade de respostas às necessidades da população do campo.

Nessa perspectiva, é importante destacar o interesse do MST em instituir Coletivos de Saúde em seus acampamentos e assentamentos, de modo a impulsionar a luta pelo Direito à Saúde em parceria com organizações não governamentais, instituições de ensino-pesquisa e profissionais da área de saúde. É notório o papel importante do Coletivo Nacional de Saúde do MST para discussão e formulação de políticas de Saúde, organização do setor de saúde dentro dos acampamentos e assentamentos e desenvolvimento de práticas educativas para/com 
as lideranças do movimento, mas verifica-se enorme lacuna de pesquisas que revelem mais profundamente as estratégias de organização e luta do MST pela saúde no Brasil.

Um aspecto a ser aprofundado são as contradições e divergências entre os militantes do MST sobre o SUS e a participação nas instâncias de controle social. Chama atenção o fato da tardia participação do MST nos processos decisórios em saúde somente após a criação do Grupo da Terra em 2005, que também coloca em questão a capacidade de o movimento sanitário incorporar os movimentos do campo em sua base de sustentação. Tais elementos precisam ser mais bem explorados, de maneira a superar a incipiente produção científica sobre as formas de mobilização e luta pela garantia do direito à saúde da população do campo, inclusive, investigando se o MST tem sido um aliado no processo de RSB.

\section{Colaboradores}

LDV Barros participou da concepção e coordenação da pesquisa, análise e interpretação dos resultados, redação do artigo; revisão do texto e aprovação da versão final do manuscrito. CF Teixeira participou da concepção do artigo, da orientação do estudo, revisão do texto e aprovação da versão final do manuscrito

\section{Referências}

1. Movimento dos Trabalhadores Rurais Sem Terra [internet]. [acesso em 2018 out 15]. Disponível em: http://www.mst.org.br.

2. Santos JCBD, Hennington EA. Aqui ninguém domina ninguém: sentidos do trabalho e produção de saúde para trabalhadores de assentamento do Movimento dos Trabalhadores Rurais Sem Terra. Cad Saúde Pública. 2013 ago; 29(8):1595-1604.

3. Stédile JP, Fernandes BM. Brava Gente: a trajetória do MST e a luta pela terra no Brasil. 2. ed. São Paulo: Perseu Abramo; 2012.
4. Severo DO, Ros MAD. A Participação no controle social do SUS: concepção do Movimento dos Trabalhadores Rurais Sem Terra. Saúde Soc. São Paulo, 2012 maio; 21 (supl. 1):177-184.

5. Paim JS. Reforma Sanitária Brasileira: Contribuição para a Compreensão e Crítica. Rio de Janeiro: Fiocruz; 2008.

6. Brasil. Constituição, (1988). Constituição da República Federativa do Brasil. Brasília: Senado Federal; 1988.

7. Paim, JS. A Constituição Cidadã e os 25 anos do Sis- 
tema Único de Saúde. Cad. Saúde Pública. 2013 out; 29(10):1927-1953.

8. Teixeira CF, Souza LE, Paim JS. Sistema Único de Saúde, a difícil construção de um sistema universal na sociedade brasileira. In: Almeida Filho N, Paim JS. Saúde Coletiva: teoria e prática. Rio de Janeiro: MEDBOOK; 2014. p. 121-137.

9. Botelho LLR, Cunha CCDA, Macedo M. O método da revisão integrativa nos estudos organizacionais. Gestão e Sociedade. 2011 maio-ago; 5(11):121-136.

10. Biblioteca Virtual de Saúde BVS [internet]. [acesso em 2018 out 15]. Disponível em: http://brasil.bvs. $\mathrm{br} /$.

11. Banco de Periódicos Capes [homepage na Internet]. [acesso em 2018 out 15]. Disponível em: http:// www.periodicos.capes.gov.br/.

12. Minayo MCDS. O desafio do conhecimento: pesquisa qualitativa em saúde. 14. ed. São Paulo: Hucitec; 2014.

13. Gomes R. A análise e interpretação de dados em pesquisa qualitativa. In: Minayo MCDS. Pesquisa Social: Teoria, Método e Criatividade. 33. ed. Petrópolis: Vozes; 2013. p. 79-108.

14. Carneiro FF, Tambellini AT, Silva JA, et al. A saúde das populações do campo: das políticas oficiais às contribuições do Movimento dos Trabalhadores Rurais Sem Terra (MST). Cad. Saúde Colet. 2007 abr-jun; 15(2):209-230.

15. Ruckert B, Machado AR, Santos CCA, et al. Diálogos entre a Saúde do Campo e a Saúde Mental: a experiência da Oficina de Educação Popular em Saúde Mental do MST na ESP MG. Interface. Botucatu, 2014; 18(supl. 2):1537-1546.

16. Ricardo LM. O uso de plantas medicinais na medicina popular praticada em assentamentos do MST do estado do Rio de Janeiro: uma contribuição para o SUS. [dissertação]. [Rio de Janeiro]: Fiocruz; 2011. $192 \mathrm{p}$.
17. Cavalcante IMDS, Nogueira LMV. Práticas sociais coletivas para a saúde no assentamento Mártires de Abril na Ilha de Mosqueiro-Belém, Pará. Esc. Anna Nery. 2008 set; 12(3):492-499.

18. Sodré F. A agenda global dos movimentos sociais. Ciênc Saúde Coletiva. 2011 mar; 6(3):1781-1791.

19. Ricardo LM, Stotz EN. Educação Popular como Método de Análise: Relações entre Medicina Popular e a "Situação-limite" Vivenciada por Trabalhadores do Movimento dos Trabalhadores Rurais Sem-Terra. Revista APS. 2012 out-dez; 15(4):435-442.

20. Fontoura Junior EE, Souza KR, Renovato RD, et al. Relações de saúde e trabalho em assentamento rural do MST na região de fronteira Brasil-Paraguai. Trab. educ. saúde. 2011 nov; 9(3):379-397.

21. Rotulo LM, Domingues RC, Silva ES, et al. O Cuidado em Saúde e a Extensão Universitária Referenciados na Educação Popular: uma História de Convivência com a Realidade do Campo. Revista APS. 2015 out-dez; 18(4):539-543.

22. Teixeira IMDC, Oliveira MW. Práticas de cuidado à saúde de mulheres camponesas. Interface. Botucatu, 2014; 18 (supl. 2):1341-1354.

23. Caldart RS. Pedagogia do Movimento Sem Terra. São Paulo: Expressão Popular; 2012.

24. Ebling SBD, Falkembach EMF, Nascimento LA, et al. As Mulheres e suas 'Lidas': Compreensões Acerca de Trabalho e Saúde. Trab. educ. saúde. 2015 dez; 13(3):581-596

25. Maeda ST, Borges ALV, Nakamura E, et al. Access to pre-natal care and cervical cancer prevention action among women from Brazilian Landless Workers Movement. Texto contexto - Enferm. 2007 set; 16(3):433-438.

26. Carneiro FF, Tambellini AT, Silva JA, et al. Saúde de famílias do Movimento dos Trabalhadores Sem Terra e de bóias-frias, Brasil, 2005. Rev. Saúde Pública. 2008 ago; 42(4):757-763. 
27. Santos JCBD. O movimento dos trabalhadores rurais sem-terra e as relações entre saúde, trabalho e ambiente em um assentamento rural no estado do Rio de Janeiro. [Dissertação]. [Rio de Janeiro]: Fiocruz; 2011. 142 p.

28. Brasil. Ministério da Saúde. Secretaria de Atenção à Saúde. Política nacional de atenção integral à saúde da mulher: princípios e diretrizes. Brasília, DF: Ministério da Saúde; 2004.

29. Brasil. Ministério da Saúde. Portaria $n^{\circ} 1.823$, de 23 de agosto de 2012. Institui a Política Nacional de Saúde do Trabalhador e da Trabalhadora. Diário Oficial da União. 24 Ago 2012.

30. Brasil. Ministério da Saúde. Portaria no 971, de 03 de maio de 2006. Aprova a Política Nacional de Práticas Integrativas e Complementares (PNPIC) no Sistema Único de Saúde. Diário Oficial da União. 4 Maio 2006.

31. Brasil. Decreto $\mathrm{n}^{\mathrm{o}} 5.813$, de 22 de junho de 2006. Aprova a Política Nacional de Plantas Medicinais e Fitoterápicos e dá outras providências. Diário Oficial da União. 23 Jun 2006.

32. Brasil. Lei $n^{\circ} 12.424$, de 16 de junho de 2011. Altera a Lei no 11.977, de 7 de julho de 2009, que dispõe sobre o Programa Minha Casa, Minha Vida - PMCMV e a regularização fundiária de assentamentos localizados em áreas urbanas, as Leis nos 10.188, de 12 de fevereiro de 2001, 6.015, de 31 de dezembro de 1973, 6.766, de 19 de dezembro de 1979, 4.591, de 16 de dezembro de $1964,8.212$, de 24 de julho de 1991 , e 10.406, de 10 de janeiro de 2002 - Código Civil; revoga dispositivos da Medida Provisória no 2.197-43, de 24 de agosto de 2001; e dá outras providências. Diário Oficial da União. 20 Jun 2011.

33. Brasil. Ministério da Saúde. Portaria $n^{0} 2.866$, de 2 de dezembro de 2011. Institui, no âmbito do Sistema Único de Saúde (SUS), a Política Nacional de Saúde Integral das Populações do Campo e da Floresta (PNSIPCF). Diário Oficial da União. 5 Dez 2011.

34. Instituto Nacional de colonização e Reforma Agrária (Brasil). Pesquisa de avaliação da qualidade dos assentamentos de reforma agrária, 2010 [internet]. [acesso em: 2018 jul 03]. Disponível em: http://pqra. incra.gov.br/.

Recebido em 15/07/2018 Aprovado em 14/09/2018

Conflito de Interesses: inexistente

Suporte Financeiro: não houve 